\title{
Human Immunodeficiency Virus and Cardiovascular Disease: Revisiting the Inflammation-Thrombosis Axis
}

\author{
Keir McCutcheon ${ }^{1}$ Pravin Manga ${ }^{10}$ \\ 1 Department of Internal Medicine, School of Clinical Medicine,
Faculty of Health Sciences, University of Witwatersrand,
Johannesburg, South Africa
}

Thromb Haemost 2022;122:476-479.

\section{Introduction}

There are more than 37 million people living with the human immunodeficiency virus (PLWH) worldwide with an estimated 2 million new infections occurring annually. Antiretroviral medication has dramatically prolonged the life expectancy of PLWH resulting in a growing population of older PLWH. Based on recent data from the Netherlands, by $2030,73 \%$ of human immunodeficiency virus (HIV)-positive patients will be older than 50 years of age. ${ }^{1}$ Despite this increasing life expectancy, there is a twofold increased risk of atherosclerotic cardiovascular disease (ASCVD) and a two- to 10 -fold increased risk of venous thromboembolism (VTE). The reasons for the increased risk of cardiovascular disease among PLWH are complex and multifactorial. In addition to a high frequency of traditional risk factors in PLWH, persistent inflammation and coagulopathy appear to contribute to the increased risk of ASCVD and VTE.

HIV-1 infects CD4+ cells, mainly T helper cells, but also macrophages and dendritic cells, and directly or indirectly destroys these cells leading to immune dysfunction and deficiency. ${ }^{2}$ HIV is able to activate pattern recognition receptors (PRRs) that promote inflammasome activation in circulating monocyte-derived dendritic cells, human monocytes, and monocyte-derived macrophages. ${ }^{3}$ Although combined antiretroviral therapy (cART) suppresses circulating viral counts, there is no complete elimination of viral latency, chronic inflammation, or immune activation. This chronic inflammation in PLWH, despite cART with viral suppression, is probably related to increased traditional risk factors and proinflammatory lipids, certain antiretroviral combinations, low levels of viral replication, coinfection with other pathogens such as cytomegalovirus, and microbial translocation (- Fig. 1). ${ }^{4}$

Contributing to the proinflammatory phenotype in PLWH is lipodystrophy and adipocyte tissue dysfunction. ${ }^{5}$ Lipodystrophy (abnormal fat redistribution) is associated with metabolic derangements, such as dyslipidemia and insulin
Address for correspondence Pravin Manga, MBBCH, PhD, Internal Medicine, Faculty of Health Sciences, University of Witwatersrand, Parktown, Johannesburg 2000, South Africa

(e-mail: pravin.manga@wits.ac.za).

resistance, and is well described following cART initiation. 5,6 It is not clear whether a particular antiretroviral therapy drug or class of drugs is responsible for the abnormal fat accumulation since lipodystrophy appears to occur to some degree with any combination, even in patients with no prior exposure to protease inhibitors. ${ }^{7,8}$ Adipocyte tissue dysfunction, which is caused by both the HIV infection and certain antiretroviral agents, promotes systemic inflammation through secretion of adipokines. ${ }^{9}$ In addition, due to chronic viral load suppression with effective cART among PLWH, there is an increasing frequency of obesity, which promotes insulin resistance and chronic inflammation through adipocyte dysfunction and lipotoxicity. ${ }^{10}$

\section{Thromboinflammation in PLWH}

There is a bidirectional relationship between thrombosis and inflammation. Circulating monocytes and tissue macrophages are able to initiate thrombosis through expression of tissue factor (TF), ${ }^{11,12}$ and coagulation intermediates, such as fibrinogen, can induce and accelerate inflammation. ${ }^{13,14}$ Furthermore, activated platelets are an important source of

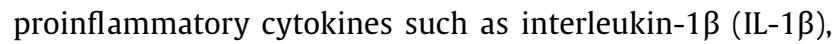
supporting their role in both coagulation and inflammation. ${ }^{15}$ Since the interaction between thrombosis and inflammation is bidirectional, chronic activation of these pathways is likely to contribute to cardiovascular morbidity and mortality in PLWH. ${ }^{13,16}$

\section{Evidence for Increased Inflammation and Thrombosis in HIV}

Markers of inflammation and thrombosis are frequently raised in PLWH regardless of viral suppression status, and are independently associated with increased morbidity and mortality. ${ }^{16}$
DOI https://doi.org/ 10.1055/s-0041-1736445. ISSN 0340-6245. (c) 2021. Thieme. All rights reserved. Georg Thieme Verlag KG,

Rüdigerstraße 14,

70469 Stuttgart, Germany 


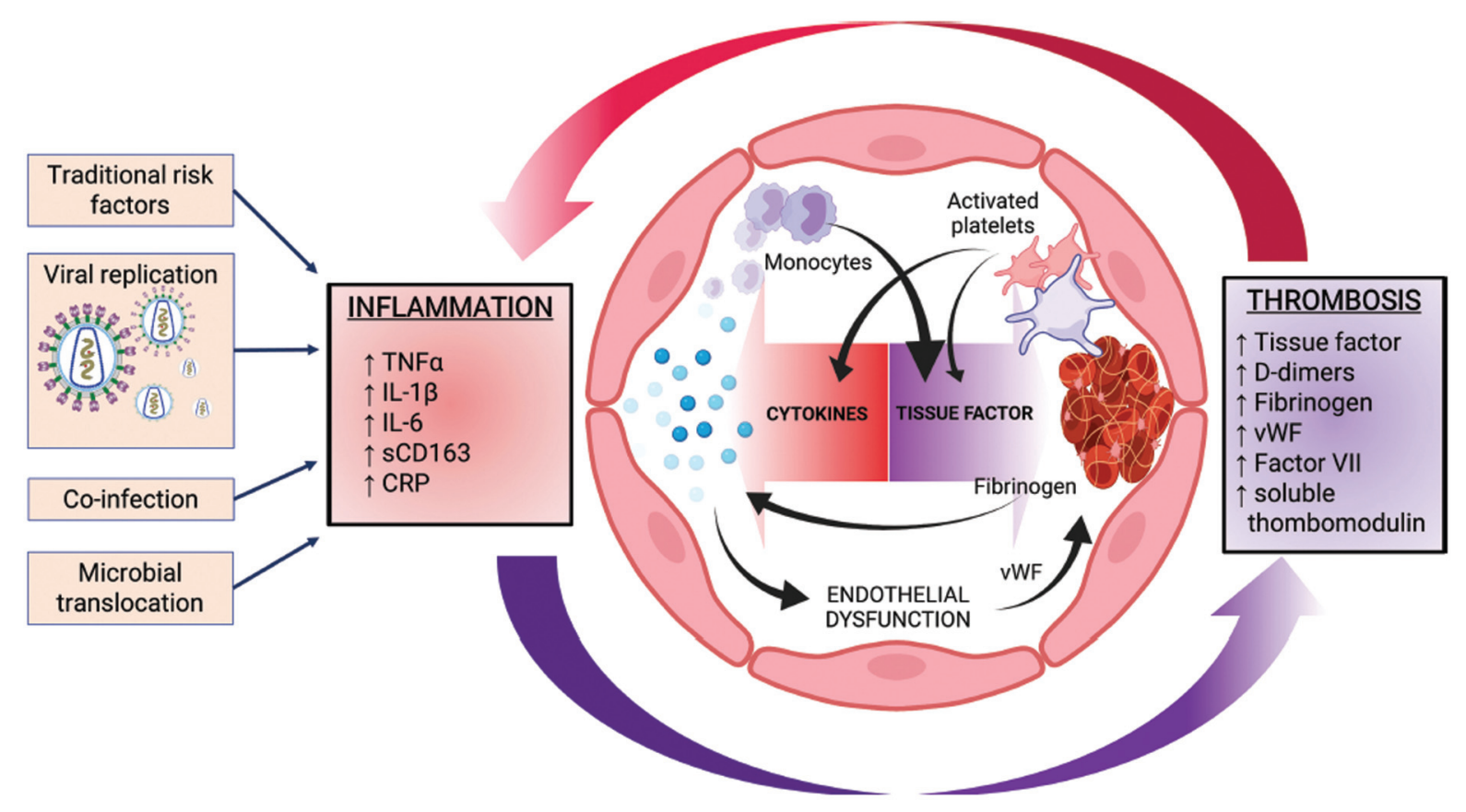

Fig. 1 Bidirectional relationship between inflammation and thrombosis in HIV-infected patients. CRP, C-reactive protein; IL-1 $\beta$, interleukin-1 $\beta$; IL6, interleukin 6; sCD163, soluble CD163; TNF- $\alpha$, tumor necrosis factor- $\alpha$; vWF, von Willebrand factor.

Serum markers of inflammation such as IL-6, tumor necrosis factor- $\alpha 1$ (TNF- $\alpha 1$ ), and TNF- $\alpha 2$, and positron emission tomography-computed tomography evidence for chronic inflammation are more prevalent in PLWH compared with HIV-negative individuals and have been shown to be associated with ASCVD. ${ }^{17}$ Soluble markers of inflammation such as sCD163 and sCXCL10, and CD14+ and CD16+ monocytes are also increased in PLWH and have been found to be independent predictors of the presence of coronary plaque. ${ }^{4,18}$

These inflammatory factors increase circulating TF, which can activate the extrinsic pathway of the coagulation cascade. Expression of TF can be induced by exposure of pericytes and fibroblasts to proinflammatory cytokines and PRRs, such as toll-like receptors, and TF is also released by circulating monocytes. It has been demonstrated that the profile of monocyte activation and TF levels in uncontrolled HIV-1 infection is similar to that of non-HIV patients with acute coronary syndromes. ${ }^{19}$ This suggests that the inflammatory and thrombotic milieu seen in patients with an acute coronary syndrome is somewhat similar in PLWH. Abnormal elevation of D-dimers, fibrinogen, factor VII, von Willebrand factor (vWF), soluble thrombomodulin, and TF levels are common in $\mathrm{PLWH}^{4}{ }^{4}$ and are independently associated with the risk for ASCVD and VTE events. ${ }^{20,21}$

\section{Coagulopathy in PLWH}

Thrombus formation requires activation of the extrinsic pathway through TF, which is expressed on all cells, or activation of the intrinsic pathway due to vessel-wall damage or endothelial dysfunction. TF expression is increased in monocytes, platelets, and plasma of PLWH, ${ }^{22}$ and is increased by several mechanisms including inflammatory cytokines and PRRs, which are induced by HIV itself or an increase in microbial products such as lipopolysaccharide (LPS) due to microbial translocation. LPS also stimulates TF expression and increases plasminogen activator inhibitor-1, which inhibits fibrinolysis thus promoting coagulation.

\section{Endothelial Dysfunction and Thromboinflammation in PLWH}

The endothelium is the interface between circulating cells, circulating factors, and the vessel wall. Endothelial cells maintain vascular health by exerting antiplatelet, antithrombotic, and anti-inflammatory actions and therefore play a critical role in the regulation of thrombosis and inflammation. ${ }^{12}$ Healthy endothelial cells secrete nitric oxide, which reduces adhesion molecule (P-selectin, E-selectin, vascular cell adhesion molecule-1 [VCAM-1], and intercellular adhesion molecule-1 [ICAM-1]) expression, thereby minimizing inflammatory cell attachment. In addition, the endothelium expresses many anticoagulant molecules such as thrombomodulin and TF pathway inhibitor. ${ }^{23}$ Chronic endothelial damage reduces the anticoagulant properties of endothelial cells by reducing the bioavailability of nitric oxide amongst others. ${ }^{12}$ The endothelium is also a major source of vWF, which mediates the initial adhesion and activation of platelets with subsequent activation of the coagulation system. ${ }^{12}$

PLWH are at increased risk of endothelial and coronary microvascular dysfunction, which is an independent risk factor for cardiovascular events. ${ }^{24,25}$ Evidence for endothelial dysfunction in PLWH has been described in several studies and is even present in young PLWH. In a prospective study of 431 youths living with perinatally acquired HIV, an increased risk of endothelial dysfunction was reported, compared with 
age-matched controls, even after adjusting for physiologic differences. ${ }^{26}$ In other studies in PLWH, elevated levels of markers of endothelial dysfunction including ICAM-1 and VCAM-1 have been described, ${ }^{27,28}$ and have been associated with thrombosis. ${ }^{15}$ The endothelial dysfunction in PLWH is likely a result of persistent inflammation, similar to other diseases associated with chronic inflammation, such as obesity, diabetes, and rheumatoid arthritis. ${ }^{29}$

\section{Platelet Activation in PLWH Contributes to Thromboinflammation}

Activated platelets promote inflammation, endothelial dysfunction, and hypercoagulability, contributing to cardiovascular events. ${ }^{30}$ In PLWH, platelets are activated by the HIV-1 virus itself, viral proteins or inflammatory molecules induced by HIV. ${ }^{15}$ Activated platelets in PLWH are able to form complexes with inflammatory $\mathrm{T}$ cells via adhesion molecules such as P-selectin. ${ }^{31}$ HIV-infected patients have high level of monocytes that express TF and P-selectin, increasing the risk for platelet-inflammatory cell complexes. ${ }^{19}$ Platelets are also responsible for the expression of TF, contributing to coagulation amplification. Ultimately, activation of the intrinsic and extrinsic pathways and amplification by platelet activation leads to fibrin deposition and thrombus formation, which is evidenced by an increase in plasma D-dimers. ${ }^{20}$

\section{Reducing the Risk of Inflammation and Thrombosis in PLWH}

Although cART decreases markers of inflammation and coagulation, these indices often do not normalize. ${ }^{32}$ Platelet hyperactivation persists even when patients are on cART. ${ }^{15}$ Therefore, interventions to reduce inflammation and thrombosis are still being sought in this population since treatment of the persistent inflammation and immune activation may lead to a reduction in the risk of ASCVD in PLWH. ${ }^{33}$

Drawing on the CANTOS (the Canakinumab Anti-inflammatory Thrombosis Outcomes Study) findings, ${ }^{34}$ the monoclonal anti-IL-1B canakinumab reduced plasma IL-6, highsensitivity C-reactive protein, and SCD163 after a single dose in 10 treated and suppressed HIV-infected patients. ${ }^{35}$ But larger studies with longer follow-up will be required. In a phase 2 randomized, double-blind, multicenter trial in 176 PLWH at increased risk for ASCVD, low-dose methotrexate had no significant effect on endothelial function or inflammatory biomarkers, but was associated with a significant decrease in CD8 + T cells. ${ }^{36}$ Several small trials are assessing the effect of antiplatelet agents on vascular surrogates, such as flow-mediated dilation. Clopidogrel has been shown to exhibit anti-inflammatory activity in addition to its antithrombotic effect in PLWH. ${ }^{37}$ Other strategies such as aspirin, factor Xa antagonists, IL-6 antagonists, janus kinase inhibitors, and adenosine reuptake inhibitors have, thus far, not yielded robust data that support the use of these agents in reducing inflammation in PLWH. ${ }^{33}$ Finally, sevelamer, a drug that lowers phosphate, has been shown to reduce factors related to ASCVD, such as soluble TF, and low-density lipoprotein cholesterol, which may have cardiovascular benefits, ${ }^{38}$ but further studies are warranted.

\section{Conclusion}

Despite viral suppression, people living with HIV on antiretroviral therapy have persistent inflammation related to several factors including traditional risk factors, adipocyte tissue dysfunction, low levels of viral replication, coinfection with other pathogens, and microbial translocation. These proinflammatory factors promote TF expression on platelets and monocytes, and promote endothelial dysfunction. This leads to a bidirectional thromboinflammatory amplification, which increases the risk of cardiovascular events such as myocardial infarction, stroke, and venous thromboembolic disease. Interventions to reduce inflammation and thrombosis in PLWH are still being actively investigated.

Conflict of Interest

None declared.

\section{References}

1 Smit M, Brinkman K, Geerlings S, et al; ATHENA observational cohort. Future challenges for clinical care of an ageing population infected with HIV: a modelling study. Lancet Infect Dis 2015;15 (07):810-818

2 Deeks SG, Overbaugh J, Phillips A, Buchbinder S. HIV infection. Nat Rev Dis Primers 2015;1:15035

3 Leal VNC, Reis EC, Pontillo A. Inflammasome in HIV infection: lights and shadows. Mol Immunol 2020;118:9-18

4 Vachiat A, McCutcheon K, Tsabedze N, Zachariah D, Manga P. HIV and ischemic heart disease. J Am Coll Cardiol 2017;69(01):73-82

5 McComsey GA, Moser C, Currier J, et al. Body composition changes after initiation of raltegravir or protease inhibitors: ACTG A5260s. Clin Infect Dis 2016;62(07):853-862

6 Worm SW, Friis-Møller N, Bruyand M, et al; D:A:D study group. High prevalence of the metabolic syndrome in HIV-infected patients: impact of different definitions of the metabolic syndrome. AIDS 2010;24(03):427-435

7 Mulligan K, Tai VW, Algren H, et al. Altered fat distribution in HIVpositive men on nucleoside analog reverse transcriptase inhibitor therapy. J Acquir Immune Defic Syndr 2001;26(05):443-448

8 Lake JE, McComsey GA, Hulgan T, et al. Switch to raltegravir from protease inhibitor or nonnucleoside reverse-transcriptase inhibitor does not reduce visceral fat in human immunodeficiency virus-infected women with central adiposity. Open Forum Infect Dis 2015;2(02):ofv059

9 Bourgeois C, Gorwood J, Olivo A, et al. Contribution of adipose tissue to the chronic immune activation and inflammation associated with HIV infection and its treatment. Front Immunol 2021; 12:670566

10 Glesby MJ, Hanna DB, Hoover DR, et al. Abdominal fat depots and subclinical carotid artery atherosclerosis in women with and without HIV infection. J Acquir Immune Defic Syndr 2018;77 (03):308-316

11 d'Alessandro E, Becker C, Bergmeier W, et al; Scientific Reviewer Committee. Thrombo-inflammation in cardiovascular disease: an expert consensus document from the third maastricht consensus conference on thrombosis. Thromb Haemost 2020;120(04):538-564

12 Verhamme P, Hoylaerts MF. The pivotal role of the endothelium in haemostasis and thrombosis. Acta Clin Belg 2006;61(05): 213-219 
13 Levi M, van der Poll T, Büller HR. Bidirectional relation between inflammation and coagulation. Circulation 2004;109(22): 2698-2704

14 Davalos D, Akassoglou K. Fibrinogen as a key regulator of inflammation in disease. Semin Immunopathol 2012;34(01):43-62

15 Pretorius E. Platelets in HIV: a guardian of host defence or transient reservoir of the virus? Front Immunol 2021;12:649465

16 Funderburg NT, Lederman MM. Coagulation and morbidity in treated HIV infection. Thromb Res 2014;133(Suppl 1):S21-S24

17 Bahrami H, Budoff M, Haberlen SA, et al. Inflammatory markers associated with subclinical coronary artery disease: the multicenter AIDS cohort study. J Am Heart Assoc 2016;5(06):5

18 Fitch KV, Srinivasa S, Abbara S, et al. Noncalcified coronary atherosclerotic plaque and immune activation in HIV-infected women. J Infect Dis 2013;208(11):1737-1746

19 Funderburg NT, Zidar DA, Shive C, et al. Shared monocyte subset phenotypes in HIV-1 infection and in uninfected subjects with acute coronary syndrome. Blood 2012;120(23):4599-4608

20 Ford ES, Greenwald JH, Richterman AG, et al. Traditional risk factors and D-dimer predict incident cardiovascular disease events in chronic HIV infection. AIDS 2010;24(10):1509-1517

21 Musselwhite LW, Sheikh V, Norton TD, et al. Markers of endothelial dysfunction, coagulation and tissue fibrosis independently predict venous thromboembolism in HIV. AIDS 2011;25(06): 787-795

22 Funderburg NT, Mayne E, Sieg SF, et al. Increased tissue factor expression on circulating monocytes in chronic HIV infection: relationship to in vivo coagulation and immune activation. Blood 2010;115(02):161-167

23 Jackson SP, Darbousset R, Schoenwaelder SM. Thromboinflammation: challenges of therapeutically targeting coagulation and other host defense mechanisms. Blood 2019;133(09):906-918

24 Torriani FJ, Komarow L, Parker RA, et al; ACTG 5152s Study Team. Endothelial function in human immunodeficiency virus-infected antiretroviral-naive subjects before and after starting potent antiretroviral therapy: The ACTG (AIDS Clinical Trials Group) Study 5152s. J Am Coll Cardiol 2008;52(07):569-576

25 Rethy L, Feinstein MJ, Sinha A, Achenbach C, Shah SJ. Coronary microvascular dysfunction in HIV: a review. J Am Heart Assoc 2020;9(01):e014018

26 Mahtab S, Zar HJ, Ntusi NAB, et al. Endothelial dysfunction in South African youth living with perinatally acquired human immunodeficiency virus on antiretroviral therapy. Clin Infect Dis 2020;71(10):e672-e679

27 Graham SM, Rajwans N, Jaoko W, et al. Endothelial activation biomarkers increase after HIV-1 acquisition: plasma vascular cell adhesion molecule-1 predicts disease progression. AIDS 2013;27 (11):1803-1813

28 Vachiat A, Dix-Peek T, Duarte R, Manga P. Endothelial dysfunction in HIV-positive patients with acute coronary syndromes. Cardiovasc J Afr 2020;31(04):58-64

29 Konst RE, Guzik TJ, Kaski JC, Maas AHEM, Elias-Smale SE. The pathogenic role of coronary microvascular dysfunction in the setting of other cardiac or systemic conditions. Cardiovasc Res 2020;116(04):817-828

30 Hauguel-Moreau M, Boccara F, Boyd A, et al. Platelet reactivity in human immunodeficiency virus infected patients on dual antiplatelet therapy for an acute coronary syndrome: the EVERE2STHIV study. Eur Heart J 2017;38(21):1676-1686

31 Green SA, Smith M, Hasley RB, et al. Activated platelet-T-cell conjugates in peripheral blood of patients with HIV infection: coupling coagulation/inflammation and T cells. AIDS 2015;29 (11):1297-1308

32 Baker JV, Huppler Hullsiek K, Bradford RL, Prosser R, Tracy RP, Key NS. Circulating levels of tissue factor microparticle procoagulant activity are reduced with antiretroviral therapy and are associated with persistent inflammation and coagulation activation among HIV-positive patients. J Acquir Immune Defic Syndr 2013;63(03):367-371

33 Titanji B, Gavegnano C, Hsue P, Schinazi R, Marconi VC. Targeting inflammation to reduce atherosclerotic cardiovascular risk in people with HIV infection. J Am Heart Assoc 2020;9(03):e014873

34 Ridker PM, Everett BM, Thuren T, et al; CANTOS Trial Group. Antiinflammatory therapy with canakinumab for atherosclerotic disease. N Engl J Med 2017;377(12):1119-1131

35 Hsue PY, Li D, Ma Y, et al. IL-1 $\beta$ inhibition reduces atherosclerotic inflammation in HIV infection. J Am Coll Cardiol 2018;72(22): 2809-2811

36 Hsue PY, Ribaudo HJ, Deeks SG, et al. Safety and impact of lowdose methotrexate on endothelial function and inflammation in individuals with treated human immunodeficiency virus: AIDS Clinical Trials Group Study A5314. Clin Infect Dis 2019;68(11): 1877-1886

37 O'Brien MP, Zafar MU, Rodriguez JC, et al. Targeting thrombogenicity and inflammation in chronic HIV infection. Sci Adv 2019;5 (06):eaav5463

38 Sandler NG, Zhang X, Bosch RJ, et al; AIDS Clinical Trials Group A5296 Team. Sevelamer does not decrease lipopolysaccharide or soluble CD14 levels but decreases soluble tissue factor, lowdensity lipoprotein (LDL) cholesterol, and oxidized LDL cholesterol levels in individuals with untreated HIV infection. J Infect Dis 2014;210(10):1549-1554 